\title{
Periodic Solution for a Stochastic Predator-Prey Model with Impulses and Holling-II Functional Response
}

\author{
Yafei Yang, Yuanfu Shao, Mengwei Li \\ College of Physics, Guilin University of Technology, Guangxi, China \\ Email:1115917086@qq.com, shaoyuanfu@163.com, 419763620@qq.com
}

How to cite this paper: Yang, Y.F., Shao, Y.F. and Li, M.W. (2019) Periodic Solution for a Stochastic Predator-Prey Model with Impulses and Holling-II Functional Response. Journal of Applied Mathematics and Physics, 7, 2212-2230.

https://doi.org/10.4236/jamp.2019.710152

Received: August 31, 2019

Accepted: October 5, 2019

Published: October 8, 2019

Copyright $\odot 2019$ by author(s) and Scientific Research Publishing Inc. This work is licensed under the Creative Commons Attribution International License (CC BY 4.0).

http://creativecommons.org/licenses/by/4.0/

\begin{abstract}
Considering the mutual interference between species, a stochastic predator-prey model with impulses and Holling-II functional response is proposed in this paper. Firstly, by constructing an equivalent system without impulses, the existence of a globally unique positive solution is proved. Secondly, in cases of the mutual coefficient $m=1$ and $0<m<1$, by constructing suitable Lyapunov functional, the existence of T-periodic solution is investigated under some certain conditions. Finally, numerical simulation is introduced to verify our main results.
\end{abstract}

\section{Keywords}

Stochastic, Impulses, Mutual Interference, Periodic Solution

\section{Introduction}

The interaction between predator and prey has long been one of the themes of mathematical biology because of the ubiquity and importance of predation. Due to the imbalance of species in the ecological environment, some species are endangered and many of them have become extinct. Therefore, protecting the diversity of ecological species has become one of the main topics in today's society. This will inspire more scholars to devote themselves to this research.

It is well known that, in the ecosystem, many factors affect the dynamics of ecological models. One of the key elements is called "functional response", which represents the consumption per unit of time. Holling-II functional response is one of the most important functional responses [1]. In the past few decades, the deterministic predator model has attracted much attention. For example, Li and Gao [2] introduced the following predator-prey system with 
Holling II functional response:

$$
\left\{\begin{array}{l}
\mathrm{d} x=x\left(r_{1}-b_{1} x-\frac{c_{1} y}{1+x}\right) \mathrm{d} t \\
\mathrm{~d} y=y\left(-r_{2}-b_{2} y+\frac{C_{2} x}{1+x}\right) \mathrm{d} t
\end{array}\right.
$$

where $x(t), y(t)$ stand for prey and predator densities at time $t$, respectively. Parameters $r_{1}, r_{2}, b_{1}, b_{2}, c_{1}$ and $c_{2}$ are positive constants; $r_{1}$ and $r_{2}$ stand for intrinsic growth rates of prey $x(t)$ and predator $y(t)$ respectively. Parameters $b_{1}$ and $b_{2}$ describe the strength of competition among individuals of species $x(t)$ or $y(t)$. Parameters $c_{1}$ and $c_{2}$ represent the capture rate of predators and the reproduction rate of converting nutrients into predators, respectively.

However, in order to better describe the phenomenon in population dynamics, Hassell initially proposed a nonlinear function of the interaction size of species. He found that as the population grew, the interference became stronger. Therefore, he introduced the concept of mutual interference constant $m \in(0,1]$ (see e.g. [3] [4] [5]). The deterministic predator-prey model with mutual interference and Holling-II function response can be expressed as

$$
\left\{\begin{array}{l}
\mathrm{d} x(t)=x(t)\left(r_{1}-b_{1} x(t)-\frac{c_{1} y^{m}(t)}{1+x}\right) \mathrm{d} t \\
\mathrm{~d} y(t)=\left(-r_{2} y(t)-b_{2} y^{2}(t)+\frac{c_{2} x(t)}{1+x} y^{m}(t)\right) \mathrm{d} t,
\end{array}\right.
$$

In recent years, system (1.2) and its various extension forms have been extensively studied by scholars (e.g. [6] [7]).

The growth of species in nature is often restricted by the environment. Because of environmental fluctuations, the parameters involved in the population model are not constant, and they may fluctuate around some average values. Based on this factor, more and more people begin to pay attention to the random population system [8] [9] [10]. We assume that environmental fluctuations mainly affect the internal growth rate $r_{1}(t)$ and the mortality $r_{2}(t)$ of predators, that is,

$$
r_{1}(t) \rightarrow r_{1}(t)+\sigma_{1}(t) B_{1}^{\prime}(t), \quad-r_{2}(t) \rightarrow-r_{2}(t)+\sigma_{2}(t) B_{2}^{\prime}(t) .
$$

where $B_{1}$ and $B_{2}$ are independent Brownian motions, $\sigma_{i}^{2}(t)(i=1,2)$ denotes the intensity of white noise. That is, we consider the following stochastic non-autonomous predator-prey system with Holling-II functional response and mutual interference:

$$
\left\{\begin{array}{l}
\mathrm{d} x(t)=x(t)\left(r_{1}-b_{1} x(t)-\frac{c_{1} y^{m}(t)}{1+x}\right) \mathrm{d} t+\sigma_{1} x(t) \mathrm{d} B_{1}(t), \\
\mathrm{d} y(t)=\left(-r_{2} y(t)-b_{2} y^{2}(t)+\frac{c_{2} x(t)}{1+x} y^{m}(t)\right) \mathrm{d} t+\sigma_{2}(t) y(t) \mathrm{d} B_{2}(t),
\end{array}\right.
$$


On the other hand, the life of species often has some big abrupt changes, such as drought, earthquake, typhoon and other big natural disasters, as well as interference from human activities, such as large-scale hunting, policy protection, etc., which will bring great changes to the number and density of species in a short period of time. Therefore, the interference of impulses to the model needs to be considered, and the following model can be established:

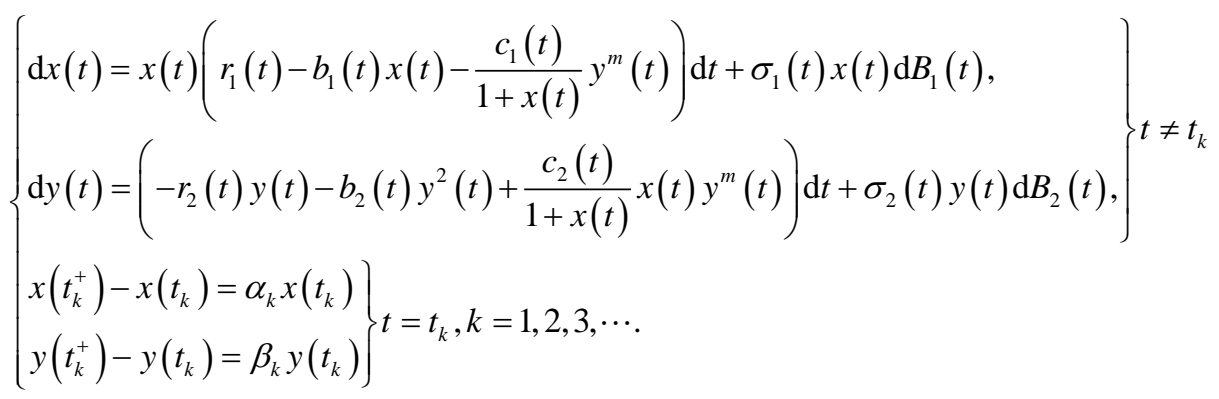

where $r_{i}(t), \quad b_{i}(t), c_{i}(t)$ and $\sigma_{i}^{2}(t)(i=1,2)$ are positive and continuous $T$-periodic functions; and the time sequences satisfies $0<t_{1}<t_{2}<t_{3}<\cdots$, and $\lim _{k \rightarrow \infty} t_{k}=+\infty$. In addition, Parameters $\alpha_{k}, \beta_{k}$ represent the impulsive effects, and $\alpha_{k}>0, \beta_{k}>0$ denote the planting of the species, and if $\alpha_{k}<0, \beta_{k}<0$, then they represent the harvest of the species. From a biological point of view, we are only looking at the positive solution of this equation. Therefore, it's a natural constraint that

$$
1+\alpha_{k}>0,1+\beta_{k}>0, k=1,2,3, \cdots .
$$

For the periodicity, we assume that there exists a positive integer $p$ such that $t_{k+p}=t_{k}+T, \alpha_{k+p}=\alpha_{k}, \beta_{k+p}=\beta_{k}, k \in Z$. Without loss of generality, we assume $[0, T) \cap\left\{t_{k}, k \in Z\right\}=\left\{t_{1}, t_{2}, t_{3} \cdots, t_{p}\right\}$.

From the biological point of view, the population density will change with the changes of some factors, such as rainfall, drought, plague, which is random. All the possible outcomes from a set $\Omega$ with typical element $w \in \Omega$. A filtration $\left\{\mathcal{F}_{t}\right\}_{t \geq 0}$ is the smallest $\sigma$-algebra $\sigma(\Omega)$, which contains $\Omega$. Throughout this paper, let $\left(\Omega,\left\{\mathcal{F}_{t}\right\}_{t \geq 0}, \mathbb{P}\right)$ is a complete probability space with a filtration $\left\{\mathcal{F}_{t}\right\}$ satisfying the usual normal conditions. (i.e., it is increasing and right continuous while $\mathcal{F}_{0}$ contains all $\mathbb{P}$-null sets), and we define $f^{u}=\sup _{t \rightarrow \infty} f(t)$, $f^{l}=\inf _{t \rightarrow \infty} f(t)$.

The main purpose of this paper is to study the existence and uniqueness of global positive periodic solutions as well as the permanence and extinction of species of system (1.4).

The rest of this paper is organized as follows. In the second part, the existence and uniqueness of global positive solution are proved. In the third part, we get that, under certain conditions, system (1.4) exists $T$-periodic solution. In the fourth part, under some conditions, we discuss the stochastic extinction and permanence of species. In the fifth part, we use numerical simulation to illustrate our results. Finally we draw a conclusion to conclude this paper. 


\section{Existence and Uniqueness of the Global Positive Solution}

Definition 2.1 ([11]). Consider the following impulsive stochastic differential equation (ISDE)

$$
\left\{\begin{array}{l}
\mathrm{d} x(t)=f(t, x(t)) \mathrm{d} t+g(t, x(t)) \mathrm{d} B(t), t \neq t_{k}, t>0, \\
x\left(t_{k}^{+}\right)-x\left(t_{k}\right)=\alpha_{k} x\left(t_{k}\right), t=t_{k}, k=1,2,3, \cdots
\end{array}\right.
$$

with the initial value

$$
x(0)=x_{0} \in \mathbb{R}^{n} .
$$

A stochastic process $x(t)=\left(x_{1}(t), x_{2}(t), \cdots, x_{n}(t)\right)^{\mathrm{T}}, t \in[0,+\infty)$ is said to be a solution of ISDE (2.1), if $x(t)$ satisfies

1) $x(t)$ is $\mathcal{F}_{t}$ adapted and is continuous on $\left(0, t_{1}\right)$ and each interval $\left(t_{k}, t_{k+1}\right), k \in \mathbb{N}$ and $f(t, x(t)) \in L^{1}\left(\mathbb{R}^{+}, \mathbb{R}^{n}\right), g(t, x(t)) \in L^{2}\left(\mathbb{R}^{+}, \mathbb{R}^{n}\right)$;

2) $x(t)$ obeys the equivalent integral equation of (2.1) for almost every $t \in \mathbb{R}_{+} \backslash t_{k}$ and satisfies the impulsive conditions at each $t \in \mathbb{R}_{+}, k \in \mathbb{N}$ a.s.;

3) For each $t_{k}, k \in \mathbb{N}, x\left(t_{k}^{+}\right)=\lim _{t \rightarrow t_{k}^{+}} x(t)$ and $x\left(t_{k}^{-}\right)=\lim _{t \rightarrow t_{k}^{-}} x(t)$ exist and $x\left(t_{k}^{-}\right)=x\left(t_{k}\right)$ with probability one.

As to the existence and uniqueness of global positive solution of system (1.4), we have the following result.

Theorem 2.1 For any initial value $\left(x_{0}, y_{0}\right) \in R_{+}^{2}$, system (1.4) has a unique global positive solution $(x(t), y(t))$ for $t \geq 0$ and the solution remains in $\mathbb{R}_{+}$with probability one.

Proof. First, we construct the following stochastic differential equation(SDE) without impulses:

$$
\left\{\begin{aligned}
\mathrm{d} y_{1}(t)= & y_{1}(t)\left(r_{1}(t)+\frac{1}{T} \sum_{j=1}^{p} \ln \left(1+\alpha_{j}\right)-b_{1}(t) A_{1}(t) y_{1}(t)\right. \\
& \left.-\frac{c_{1}(t)}{1+A_{1}(t) y_{1}(t)} A_{2}^{m}(t) y_{2}^{m}(t)\right) \mathrm{d} t+\sigma_{1}(t) y_{1}(t) \mathrm{d} B_{1}(t), \\
\mathrm{d} y_{2}(t)= & y_{2}(t)\left(-r_{2}(t)+\frac{1}{T} \sum_{j=1}^{p} \ln \left(1+\beta_{j}\right)-b_{2}(t) A_{2}(t) y_{2}(t)\right. \\
& \left.+\frac{c_{2}(t)}{1+A_{1}(t) y_{1}(t)} A_{1}(t) A_{2}^{m-1}(t) y_{1}(t) y_{2}^{m-1}(t)\right) \mathrm{d} t+\sigma_{2}(t) y_{2}(t) \mathrm{d} B_{2}(t),
\end{aligned}\right.
$$

with the initial value $\left(y_{1}(0), y_{2}(0)=\left(x_{0}, y_{0}\right)\right)$. According to the classic theory of SDE without impulse, $\operatorname{SDE}(2.2)$ has a unique global positive solution $y(t)=\left(y_{1}(t), y_{2}(t)\right) \quad$ (more details see [11]). Let $x(t)=A_{1}(t) y_{1}(t)$, $y(t)=A_{2}(t) y_{2}(t)$, then we claim that $(x(t), y(t))$ is the solution of the system (1.4).

In fact, it is easy to check that $x(t)$ and $y(t)$ are continuous on $\left(0, t_{1}\right)$ and $\left(t_{k}, t_{k+1}\right) \subset[0,+\infty), k \in N$, and for $t \neq t_{k}$, we have 


$$
\begin{aligned}
\mathrm{d} x(t)= & A_{1}^{\prime}(t) y_{1}(t) \mathrm{d} t+A_{1}(t) \mathrm{d} y_{1}(t) \\
= & A_{1}(t) y_{1}(t)\left[r_{1}(t)-b_{1}(t) A_{1}(t) y_{1}(t)-\frac{c_{1}(t)}{1+A_{1}(t) y_{1}(t)} A_{2}^{m} y_{2}^{m}\right] \mathrm{d} t \\
& +\sigma_{1}(t) A_{1}(t) y_{1}(t) \mathrm{d} w_{1}(t) .
\end{aligned}
$$

Similarly, we have

$\mathrm{d} y(t)=y(t)\left[-r_{2}(t)-b_{2}(t) y(t)+\frac{c_{2}(t)}{1+x(t)} x(t) y^{m-1}(t)\right] \mathrm{d} t-\sigma_{2}(t) y(t) \mathrm{d} w_{2}(t)$.

And for every $k \in N$,

$$
\begin{gathered}
x\left(t_{k}^{+}\right)=\lim _{t \rightarrow t_{k}^{+}} A_{1}(t) y_{1}(t)=\left(\prod_{j=1}^{p}\left(1+\alpha_{j}\right)\right)^{-\frac{t_{k}}{T}} \prod_{0 \leq t_{j} \leq t_{k}}\left(1+\alpha_{j}\right) y_{1}\left(t_{k}^{+}\right)=\left(1+\alpha_{j}\right) x\left(t_{k}\right), \\
x\left(t_{k}^{-}\right)=\lim _{t \rightarrow t_{k}^{-}} A_{1}(t) y_{1}(t)=A_{1}\left(t_{k}\right) y_{1}\left(t_{k}^{-}\right)=x\left(t_{k}\right) .
\end{gathered}
$$

In the same way, we have

$$
y\left(t_{k}^{+}\right)=\left(1+\beta_{j}\right) y\left(t_{k}\right), \quad y\left(t_{k}^{-}\right)=y\left(t_{k}\right) .
$$

This completes the proof.

\section{Existence of Positive T-Periodic Solution}

In this section, we give the existence of the positive periodic solution of the stochastic system (1.4) with impulses. For convenience of readers, we first give the definition of the periodic solution of the impulsive stochastic differential equation in the sense of distribution and the results of the existence of periodic solutions (see [12] [13]).

Definition 3.1 ([13]). A stochastic process $\xi(t)=\xi(t, \omega)$ is said to be periodic with period $T$, if for every finite sequence of numbers $t_{1}, t_{2}, \cdots, t_{n}$, the joint distribution of random variables $\xi\left(t_{1}+h\right), \xi\left(t_{2}+h\right), \cdots, \xi\left(t_{n}+h\right)$ is independent of $h$, where $h=k T \quad(k= \pm 1, \pm 2, \cdots)$.

Consider the following periodic stochastic differential equation without impulse:

$$
\mathrm{d} X(t)=f(t, X(t)) \mathrm{d} t+g(t, X(t)) \mathrm{d} B(t), t \geq 0,
$$

where $g(t, X(t))_{n \times l}$ is a $n \times l$ matrix function, $f(t, X(t))$ and the matrix $g(t, X(t))_{n \times l}$ are $T$-periodic in $t$. Then, Itô's formula can be applied to $F(t, X)$ where $X$ satisfies (3.1). This yields the stochastic differential for $F$ of the form

$$
\begin{aligned}
\mathrm{d} F(t, X(t))= & \left(\frac{\partial F(t, X)}{\partial t}+f(t, X) \frac{\partial F(t, X)}{\partial x}+\frac{1}{2} g^{2}(t, X) \frac{\partial^{2} F(t, X)}{\partial x^{2}}\right) \mathrm{d} t \\
& +g(t, X) \frac{\partial F(t, X)}{\partial x} \mathrm{~d} W(t) .
\end{aligned}
$$

Lemma 3.1 ([14]). Assume that system (3.1) has a global solution, and there exists a $T$-periodic function $V(x, t)$ such that the following conditions hold: 
1) $L V(t, x) \leq-1$ on the outside of some compact set, where

$$
L V(t, x)=\frac{\partial V}{\partial t}+\frac{\partial V}{\partial x} f+\frac{1}{2} \frac{\partial^{2} V}{\partial x^{2}} g^{2} \text {. }
$$

2) $\inf _{|x|>R} \rightarrow \infty$, as $R \rightarrow \infty$.

Then (3.1) has a $T$-periodic solution.

According to Lemma 3.1, we can obtain the main result in this section. Firstly, we can translate system (1.4) into the following two cases:

Case I. When $m=1$, we have

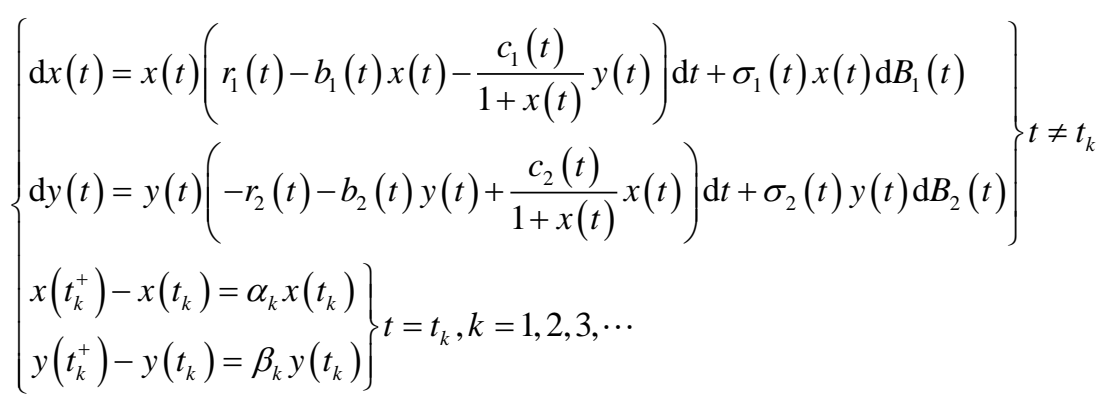

Theorem 3.1 Assume that the following assumption hold

(H1): $c_{1}^{u} A_{2}^{u}>c_{1}(t) A_{2}(t)$,

$$
\begin{aligned}
\lambda= & \frac{1}{T} \int_{0}^{T} \frac{c_{2}^{l}}{r_{1}^{u}+b_{1}^{u} A_{1}^{u}}\left(r_{1}(t)-\frac{\sigma_{1}^{2}(t)}{2}\right)-\left(r_{2}(t)+\frac{\sigma_{2}^{2}(t)}{2}\right) \mathrm{d} t \\
& +\frac{1}{T} \sum_{j=1}^{p} \ln \left(1+\alpha_{j}\right)+\frac{1}{T} \sum_{j=1}^{p} \ln \left(1+\beta_{j}\right)>0 .
\end{aligned}
$$

Then system (3.2) has a positive $T$-periodic solution.

Proof. We only need to prove the existence of a periodic solution of the equivalent system (3.3) without impulses as follows:

$$
\left\{\begin{aligned}
\mathrm{d} y_{1}(t)= & y_{1}(t)\left(r_{1}(t)+\frac{1}{T} \sum_{j=1}^{p} \ln \left(1+\alpha_{j}\right)-b_{1}(t) A_{1}(t) y_{1}(t)\right. \\
& \left.-\frac{c_{1}(t)}{1+A_{1}(t) y_{1}(t)} A_{2}(t) y_{2}(t)\right) \mathrm{d} t+\sigma_{1}(t) y_{1}(t) \mathrm{d} B_{1}(t), \\
\mathrm{d} y_{2}(t)= & y_{2}(t)\left(-r_{2}(t)+\frac{1}{T} \sum_{j=1}^{p} \ln \left(1+\beta_{j}\right)-b_{2}(t) A_{2}(t) y_{2}(t)\right. \\
& \left.+\frac{c_{2}(t)}{1+A_{1}(t) y_{1}(t)} A_{1}(t) y_{1}(t)\right) \mathrm{d} t+\sigma_{2}(t) y_{2}(t) \mathrm{d} B_{2}(t),
\end{aligned}\right.
$$

The global existence of the solution has been ensured by Theorem 2.1. Then, we only have to verify the conditions of Lemma 3.1.

Define a $C^{2}$-function $V(t, x, y): \mathbb{R}_{+}^{2} \rightarrow \mathbb{R}_{+}$as follows:

$$
\begin{aligned}
& V(x, y, t) \\
& =M\left[-\ln y+\frac{c_{2}^{l}}{r_{1}^{u}+b_{1}^{u} A_{1}^{u}}\left(-\ln \left(\frac{x}{1+x}\right)+\frac{c_{1}^{u} A_{2}^{u}}{r_{2}^{l}} y\right)\right]+\frac{(x+p y)^{\theta+1}}{\theta+1}+M W(t)(3.5) \\
& =V_{1}(x, y)+V_{2}(x, y)+V_{3}(t)
\end{aligned}
$$


where $p=\left(\frac{c_{1}^{l}}{c_{2}^{u}}\right), \theta$ is a constant such that $0<\theta<\min \left\{1, \frac{2 r_{2}^{l}}{\left(\sigma_{1}^{u}\right)^{2}}\right\}$. Parameter $M>0$ will be given later and $W(t)$ satisfies

$$
W^{\prime}(t)=-\lambda+\frac{c_{2}^{l}}{r_{1}^{u}+b_{1}^{u} A_{1}^{u}}\left(r_{1}(t)-\frac{\sigma_{1}^{2}(t)}{2}\right)-\left(r_{2}(t)+\frac{\sigma_{2}^{2}(t)}{2}\right) .
$$

It is easy to check that $W(t)$ is a $T$-periodic function. Indeed

$$
\begin{aligned}
& W(t+T)-W(t) \\
&=\int_{t}^{t+T} W^{\prime}(s) \mathrm{d} s \\
&=-\int_{0}^{T}\left[\frac{c_{2}^{l}}{r_{1}^{u}+b_{1}^{u} A_{1}^{u}}\left(r_{1}(t)-\frac{\sigma_{1}^{2}(t)}{2}\right)-\left(r_{2}(t)+\frac{\sigma_{2}^{2}(t)}{2}\right)\right] \mathrm{d} s \\
&+\int_{T}^{t+T}\left[\frac{c_{2}^{l}}{r_{1}^{u}+b_{1}^{u} A_{1}^{u}}\left(r_{1}(t)-\frac{\sigma_{1}^{2}(t)}{2}\right)-\left(r_{2}(t)+\frac{\sigma_{2}^{2}(t)}{2}\right)\right] \mathrm{d} s \\
&= 0 .
\end{aligned}
$$

According to the periodicity of $r_{i}(t), \sigma_{i}(t), i=1,2, W(t)$ is a $T$-periodic function. To verify condition (2) of Lemma 3.1, we only need to show that

$\inf _{(t, x, y) \in[0,+\infty) \times\left(R^{2} \backslash U_{k}\right)} V(t, x, y) \rightarrow \infty \mathrm{m}$ as $k \rightarrow \infty$.

Here, $U_{k}=\left(\frac{1}{k}, k\right) \times\left(\frac{1}{k}, k\right)$. All the coefficients of the quadratic term in $V(t, x, y)$ are positive, thus, condition (2) of Lemma 3.1 is satisfied.

Next we prove condition (1) of Lemma 3.1. By the Itô's formula (3.2), we have

$$
\begin{aligned}
L(-\ln y) & =r_{2}(t)-\frac{1}{T} \sum_{j=1}^{p} \ln \left(1+\beta_{j}\right)+b_{2}(t) A_{2}(t) y-\frac{c_{2}(t) x A_{1}(t)}{1+x A_{1}(t)}+\frac{\sigma_{2}^{2}(t)}{2}, \\
L\left(-\ln \left(\frac{x}{1+x}\right)\right) & \\
= & -\frac{1}{1+x}\left[r_{1}(t)+\frac{1}{T} \sum_{j=1}^{p} \ln \left(1+\alpha_{j}\right)-b_{1}(t) A_{1}(t) x-\frac{c_{1}(t) y A_{2}(t)}{1+x A_{1}(t)}\right] \\
& +\frac{\sigma_{1}^{2}(t)\left(1+x-x^{2}\right)}{2(1+x)^{2}} \\
\leq & -\left[r_{1}(t)+\frac{1}{T} \sum_{j=1}^{p} \ln \left(1+\alpha_{j}\right)-\frac{\sigma_{1}^{2}(t)}{2}\right]+\frac{x}{1+x}\left(r_{1}(t)+b_{1}(t) A_{1}(t)\right) \\
& -\frac{\sigma_{1}^{2}(t) x^{2}}{2}+\frac{c_{1}(t) y A_{2}(t)}{1+x A_{1}(t)} \frac{1}{1+A_{1}(t) x} \\
\leq & -\left[r_{1}(t)+\frac{1}{T} \sum_{j=1}^{p} \ln \left(1+\alpha_{j}\right)-\frac{\sigma_{1}^{2}(t)}{2}\right]+c_{1}(t) y A_{2}(t) \\
+ & \frac{x}{1+x}\left[r_{1}^{u}+b_{1}^{u} A_{1}^{u}+\frac{1}{T} \sum_{j=1}^{p} \ln \left(1+\alpha_{j}\right)\right] .
\end{aligned}
$$

Using (3.6), (H1) and (3.7), we have 


$$
\begin{aligned}
L V_{1}(x, y) \leq & M\left[r_{2}(t)-\frac{1}{T} \sum_{j=1}^{p} \ln \left(1+\beta_{j}\right)+b_{2}(t) A_{2}(t) y+\frac{\sigma_{2}^{2}(t)}{2}\right. \\
& \left.-\frac{c_{2}^{l}}{r_{1}^{u}+b_{1}^{u} A_{1}^{u}}\left(r_{1}(t)+\frac{1}{T} \sum_{j=1}^{p} \ln \left(1+\alpha_{j}\right)-\frac{\sigma_{1}^{2}(t)}{2}\right)\right] \\
& +M q\left(\frac{1}{T} \sum_{j=1}^{p} \ln \left(1+\beta_{j}\right) y+\frac{c_{1}(t) x y}{1+x}\right) \\
& +M \frac{c_{2}^{l}}{r_{1}^{u}+b_{1}^{u} A_{1}^{u}} \frac{1}{T} \sum_{j=1}^{p} \ln \left(1+\alpha_{j}\right)
\end{aligned}
$$

where $q=\frac{c_{2}^{l}}{r_{1}^{u}+b_{1}^{u} A_{1}^{u}} \times \frac{c_{1}^{u} A_{2}^{u}}{r_{2}^{l}}$. From (3.5) and (3.8), we have

$$
\begin{aligned}
& L V_{1}+L V_{3} \\
& \leq M\left[r_{2}(t)-\frac{1}{T} \sum_{j=1}^{p} \ln \left(1+\beta_{j}\right)+\frac{\sigma_{2}^{2}(t)}{2}-\frac{c_{2}^{l}}{r_{1}^{u}+b_{1}^{u} A_{1}^{u}}\left(r_{1}(t)+\frac{1}{T} \sum_{j=1}^{p} \ln \left(1+\alpha_{j}\right)-\frac{\sigma_{1}^{2}(t)}{2}\right)\right] \\
& +M q\left(\frac{1}{T} \sum_{j=1}^{p} \ln \left(1+\beta_{j}\right) y+\frac{c_{1}(t) x y}{1+x}\right)+M \frac{c_{2}^{l}}{r_{1}^{u}+b_{1}^{u} A_{1}^{u}}\left[r_{1}+\frac{1}{T} \sum_{j=1}^{p} \ln \left(1+\alpha_{j}\right)-\frac{\sigma_{1}^{2}(t)}{2}\right] \\
& -M \lambda-M\left[r_{2}-\frac{1}{T} \sum_{j=1}^{p} \ln \left(1+\beta_{j}\right)+\frac{\sigma_{2}^{2}(t)}{2}\right]+M \frac{c_{2}^{l}}{r_{1}^{u}+b_{1}^{u} A_{1}^{u}} \frac{1}{T} \sum_{j=1}^{p} \ln \left(1+\alpha_{j}\right) \\
& \leq-M \lambda+M q \frac{c_{1}^{u} x y}{1+x}+C
\end{aligned}
$$

Here, $C=M q \frac{1}{T} \sum_{j=1}^{p} \ln \left(1+\beta_{j}\right) y+M \frac{c_{2}^{l}}{r_{1}^{u}+b_{1}^{u} A_{1}^{u}} \frac{1}{T} \sum_{j=1}^{p} \ln \left(1+\alpha_{j}\right)$, and

$$
\begin{aligned}
L & V_{2}(x, y) \\
= & (x+p y)^{\theta}\left[r_{1}(t) x+\frac{1}{T} \sum_{j=1}^{p} \ln \left(1+\alpha_{j}\right) x-b_{1}(t) A_{1}(t) x^{2}+\frac{1}{T} \sum_{j=1}^{p} \ln \left(1+\beta_{j}\right) y\right. \\
& \left.-p^{2} b_{2}(t) y^{2}-p r_{2}(t) y+\frac{-c_{1}(t)+p c_{2}(t)}{1+x A_{1}(t)} x y A_{1}(t) A_{2}(t)\right] \\
& +\frac{\theta}{2}(x+p y)^{\theta-1}\left[\sigma_{1}^{2}(t) x^{2}+p^{2} \sigma_{2}^{2}(t) y^{2}\right] \\
\leq & (x+p y)^{\theta} r_{1}^{u} x-b_{1}^{l} A_{1}^{l} x^{2+\theta}-p^{1+\theta} r_{2}^{l} y^{1+\theta}-p^{2+\theta} b_{2}^{l} y^{2+\theta}+\frac{\theta}{2} p^{1+\theta}\left(\sigma_{2}^{u}\right)^{2} y^{1+\theta} \\
& +(x+p y)^{\theta}\left[\frac{1}{T} \sum_{j=1}^{p} \ln \left(1+\alpha_{j}\right) x+\frac{1}{T} \sum_{j=1}^{p} \ln \left(1+\beta_{j}\right) y\right]+\frac{\theta}{2}\left(\sigma_{1}^{u}\right)^{2} x^{1+\theta} \\
\leq & -\frac{b_{1}^{l} A_{1}^{l} x^{2+\theta}}{2}-\frac{p^{1+\theta}}{2}\left(r_{2}^{l}-\frac{\theta}{2}\left(\sigma_{2}^{u}\right)^{2}\right) y^{1+\theta}+C_{1} \\
& C_{1}=\sup _{(x, y) \in R_{+}^{2}}\left\{-\frac{b_{1}^{l} A_{1}^{l} x^{2+\theta}}{2}-\frac{p^{1+\theta}}{2}\left(r_{2}^{l}-\frac{\theta}{2}\left(\sigma_{2}^{u}\right)^{2}\right) y^{1+\theta}+(x+p y)^{\theta} r_{1}^{u} x\right.
\end{aligned}
$$

where

$$
\left.+\frac{\theta}{2}\left(\sigma_{1}^{u}\right)^{2} x^{1+\theta}+(x+p y)^{\theta}\left[\frac{1}{T} \sum_{j=1}^{p} \ln \left(1+\alpha_{j}\right) x+\frac{1}{T} \sum_{j=1}^{p} \ln \left(1+\beta_{j}\right) y\right]\right\},
$$


and in (3.11), we use $(x+p y)^{\theta} \leq x^{\theta},(x+p y)^{\theta} \leq p^{\theta} y^{\theta}$ in the second inequality. Then we have

$$
\begin{aligned}
L V(t, x, y) & =L V_{1}+L V_{2}+L V_{3} \\
& \leq-M \lambda+M q \frac{c_{1}^{u} x y}{1+x}-\frac{b_{1}^{l} A_{1}^{l} x^{2+\theta}}{2}-\frac{p^{1+\theta}}{2}\left(r_{2}^{l}-\frac{\theta}{2}\left(\sigma_{2}^{u}\right)^{2}\right) y^{1+\theta}+C_{2} .
\end{aligned}
$$

where $C_{2}=C+C_{1}$.

$$
\text { Let } M=\frac{2}{\lambda} \max \left\{2, \sup _{(x, y) \in R_{+}^{2}}\left\{-\frac{b_{1}^{l} A_{1}^{l} x^{2+\theta}}{4}-\frac{p^{1+\theta}}{4}\left(r_{2}^{l}-\frac{\theta}{2}\left(\sigma_{2}^{u}\right)^{2}\right) y^{1+\theta}+C_{2}\right\}\right\} \text {. }
$$

To confirm the condition (1) of Lemma 3.2, we choose a sufficiently small constant $\varepsilon$ such that:

$$
\begin{gathered}
0<\varepsilon \leq\left\{\frac{\lambda}{4 q}, \frac{p^{1+\theta}}{4 M q}\left(r_{2}^{l}-\frac{\theta}{2}\left(\sigma_{2}^{u}\right)^{2}\right), \frac{b_{1}^{l} A_{1}^{l}}{4 M q}\right\}, \\
-M \lambda-\frac{b_{1}^{l} A_{1}^{l}}{4 \varepsilon^{2+\theta}}+C_{3} \leq-1, \\
-M \lambda-\frac{p^{1+\theta}}{4 \varepsilon^{2+\theta}}\left(r_{2}^{l}-\frac{\theta}{2}\left(\sigma_{2}^{u}\right)^{2}\right)+C_{4} \leq-1 .
\end{gathered}
$$

Define a bounded closed set as follows:

$$
D=\left\{(x, y) \in R_{+}^{2}: \varepsilon \leq x \leq \frac{1}{\varepsilon}, \varepsilon \leq y \leq \frac{1}{\varepsilon}\right\} .
$$

Denote

$$
\begin{array}{cc}
D_{\varepsilon}^{1}=\left\{(x, y) \in R_{+}^{2} \mid 0<x \leq \varepsilon\right\}, & D_{\varepsilon}^{2}=\left\{(x, y) \in R_{+}^{2} \mid 0<y \leq \varepsilon\right\}, \\
D_{\varepsilon}^{3}=\left\{(x, y) \in R_{+}^{2} \mid x \geq \frac{1}{\varepsilon}\right\}, & D_{\varepsilon}^{4}=\left\{(x, y) \in R_{+}^{2} \mid y \geq \frac{1}{\varepsilon}\right\} .
\end{array}
$$

Clearly, $D_{\varepsilon}^{C}=D_{\varepsilon}^{1} \cup D_{\varepsilon}^{2} \cup D_{\varepsilon}^{3} \cup D_{\varepsilon}^{4}$.

Now we prove $L V(t, x, y) \leq-1$ on each domain.

Case 1 . If $(t, x, y) \in[0,+\infty) \times D_{\varepsilon}^{1}$, then it is easy to verify that

$$
\begin{aligned}
\frac{x y}{1+x} & \leq x y \leq \varepsilon y \leq \varepsilon\left(1+y^{1+\theta}\right) \text {, and } \\
& L V(t, x, y) \\
& \leq-\frac{M \lambda}{4}-\frac{b_{1}^{l} A_{1}^{l} x^{2+\theta}}{4}+\left[-\frac{M \lambda}{4}+M q \varepsilon\right]+\left[-\frac{p^{1+\theta}}{4}\left(r_{2}^{l}-\frac{\theta}{2}\left(\sigma_{2}^{u}\right)^{2}\right)+M q \varepsilon\right] y^{1+\theta} \\
& +\left[-\frac{M \lambda}{2}+\sup _{(x, y) \in R_{+}^{2}}\left\{-\frac{b_{1}^{l} A_{1}^{l} x^{2+\theta}}{4}-\frac{p^{1+\theta}}{4}\left(r_{2}^{l}-\frac{\theta}{2}\left(\sigma_{2}^{u}\right)^{2}\right) y^{1+\theta}+C_{2}\right\}\right] .
\end{aligned}
$$

Combining with the definition of $\frac{M \lambda}{4} \geq 1$ and (3.13), we have

$$
L V(t, x, y) \leq-\frac{M \lambda}{4}-\frac{b_{1}^{l} A_{1}^{l} x^{2+\theta}}{4} \leq-\frac{M \lambda}{4} \leq-1,
$$

Thus, $L V(t, x, y) \leq-1$ for al $(x, y) \in D_{\varepsilon}^{1}$. 
Case 2. Similarly, for any $(x, y) \in D_{\varepsilon}^{2}$, owing to $\frac{x y}{1+x} \leq x y \leq \varepsilon x \leq \varepsilon\left(1+x^{2+\theta}\right)$, we have that

$$
\begin{aligned}
& L V(t, x, y) \\
& \leq-\frac{M \lambda}{4}+\left[-\frac{M \lambda}{4}+M q \varepsilon\right]-\frac{p^{1+\theta}}{4}\left(r_{2}^{l}-\frac{\theta}{2}\left(\sigma_{2}^{u}\right)^{2}\right) y^{1+\theta}+\left[-\frac{b_{1}^{l} A_{1}^{l}}{4}+\varepsilon M q\right] x^{2+\theta} \\
& +\left[-\frac{M \lambda}{2}+\left\{\sup _{(x, y) \in R_{+}^{2}}-\frac{b_{1}^{l} A_{1}^{l}}{4} x^{2+\theta}-\frac{p^{1+\theta}}{4}\left(r_{2}^{l}-\frac{\theta}{2}\left(\sigma_{2}^{u}\right)^{2}\right) y^{1+\theta}+C_{2}\right\}\right] .
\end{aligned}
$$

Together with (3.13), we can also get

$$
L V(t, x, y) \leq-\frac{M \lambda}{4}-\frac{p^{1+\theta}}{4}\left(r_{2}^{l}-\frac{\theta}{2}\left(\sigma_{2}^{u}\right)^{2}\right) y^{1+\theta} \leq-\frac{M \lambda}{4} \leq-1 .
$$

Thus, $L V(t, x, y) \leq-1$ for all $(x, y) \in D_{\varepsilon}^{2}$.

Case 3. For any $(x, y) \in D_{\varepsilon}^{3}$, since $\frac{x y}{1+x} \leq y$, it is easy to have

$$
\begin{aligned}
& \operatorname{LV}(t, x, y) \\
& \leq-M \lambda-\frac{b_{1}^{l} A_{1}^{l}}{4 \varepsilon^{2+\theta}}+\left[-\frac{b_{1}^{l} A_{1}^{l}}{4} x^{2+\theta}-\frac{p^{1+\theta}}{2}\left(r_{2}^{l}-\frac{\theta}{2}\left(\sigma_{2}^{u}\right)^{2}\right) y^{1+\theta}+M q \frac{c_{1}^{u} x y}{1+x}+C_{2}\right] \\
& \leq-M \lambda-\frac{b_{1}^{l} A_{1}^{l}}{4 \varepsilon^{2+\theta}}+C_{3} .
\end{aligned}
$$

where $C_{3}=\sup _{(x, y) \in R_{+}^{2}}\left\{-\frac{b_{1}^{l} A_{1}^{l}}{4} x^{2+\theta}-\frac{p^{1+\theta}}{2}\left(r_{2}^{l}-\frac{\theta}{2}\left(\sigma_{2}^{u}\right)^{2}\right) y^{1+\theta}+M q \frac{c_{1}^{u} x y}{1+x}+C_{2}\right\}$, and we get $L V(t, x, y) \leq-1$ in this domain from (3.14).

Case 4. Similarly, for any $(x, y) \in D_{\varepsilon}^{4}$, we have

$$
\begin{aligned}
& L V(t, x, y) \\
\leq & -M \lambda-\frac{p^{1+\theta}}{4 \varepsilon^{2+\theta}}\left(r_{2}^{l}-\frac{\theta\left(\sigma_{2}^{u}\right)^{2}}{2}\right)-\frac{p^{1+\theta}}{4}\left(r_{2}^{l}-\frac{\theta\left(\sigma_{2}^{u}\right)^{2}}{2}\right) y^{1+\theta} \\
& -\frac{b_{1}^{l} A_{1}^{l}}{2} x^{2+\theta}+M q \frac{c_{1}^{u} x y}{1+x}+C_{2} \\
\leq & -M \lambda-\frac{p^{1+\theta}}{4 \varepsilon^{2+\theta}}\left(r_{2}^{l}-\frac{\theta}{2}\left(\sigma_{2}^{u}\right)^{2}\right)+C_{4} .
\end{aligned}
$$

where $\quad C_{4}=\sup _{(x, y) \in R_{+}^{2}}\left\{-\frac{b_{1}^{l} A_{1}^{l}}{2} x^{2+\theta}-\frac{p^{1+\theta}}{4}\left(r_{2}^{l}-\frac{\theta}{2}\left(\sigma_{2}^{u}\right)^{2}\right) y^{1+\theta}+M q \frac{c_{1}^{u} x y}{1+x}+C_{2}\right\}$.

It is clear that $L V(t, x, y) \leq-1$ in this domain.

Therefore

$L V(t, x, y) \leq-1$, for all $(t, x, y) \in[0,+\infty) \times D^{C}$.

That is to say, Condition (1) of Lemma 3.1 is verified. Thus system (3.2) has a $T$-periodic solution. This completes the proof.

Now we're going to talk about another case. 
Case II. If $0<m<1$, then we have

$$
\left\{\begin{array}{l}
\mathrm{d} x(t)=x(t)\left(r_{1}(t)-b_{1}(t) x(t)-\frac{c_{1}(t)}{1+x(t)} y^{m}(t)\right) \mathrm{d} t+\sigma_{1}(t) x(t) \mathrm{d} B_{1}(t), \\
\mathrm{d} y(t)=y(t)\left(-r_{2}(t)-b_{2}(t) y(t)+\frac{c_{2}(t)}{1+x(t)} x(t) y^{m-1}(t)\right) \mathrm{d} t+\sigma_{2}(t) y(t) \mathrm{d} B_{2}(t), \\
x\left(t_{k}^{+}\right)-x\left(t_{k}\right)=\alpha_{k} x\left(t_{k}\right) \\
y\left(t_{k}^{+}\right)-y\left(t_{k}\right)=\beta_{k} y\left(t_{k}\right)
\end{array}\right\} t=t_{k}, k=1,2,3, \cdots .
$$

Theorem 3.3 Assume that the following assumption holds

$$
\begin{aligned}
\lambda_{1}= & \frac{1}{T} \int_{0}^{T}\left(r_{1}(t)-\frac{\sigma_{1}^{2}(t)}{2}\right) \mathrm{d} t-\frac{1}{M T} \int_{0}^{T}\left(r_{2}(t)+\frac{\sigma_{2}^{2}(t)}{2}\right) \mathrm{d} t \\
& +\frac{1}{T} \sum_{j=1}^{p} \ln \left(1+\alpha_{j}\right)-\frac{1}{T} \sum_{j=1}^{p} \ln \left(1+\beta_{j}\right)>0
\end{aligned}
$$

Then system (3.16) has a positive $T$-periodic solution.

Proof. We only need to prove the existence of a periodic solution of the equivalent system (2.2) without impulses. The global existence of the solution has been ensured by Theorem 2.1. Then, we only have to verify the conditions of Lemma 3.1.

Define a $C^{2}$-function $V_{4}(t, x, y): \mathbb{R}_{+}^{2} \rightarrow \mathbb{R}_{+}$

$$
\begin{aligned}
& V_{4}(t, x, y) \\
& =M_{1}\left[-\frac{b_{1}^{l}\left(A_{2}^{1-m}\right)^{l}}{c_{2}^{u}} \frac{y^{1-m}}{1-m}-\ln x+\frac{H A_{2}^{u}}{b_{2}^{l}} y\right]-\ln y+\frac{c_{2}^{u}}{c_{1}^{l}} y+A_{1}^{u} x+M_{1} W_{1}(t) \\
& =V_{5}(x, y)+V_{6}(t)
\end{aligned}
$$

Here, $H>0$ will be given in (3.18), and $W_{1}(t)$ is $T$-periodic. Same as case I, so the condition (2) of Lemma 3.1 is satisfied.

Next, by the Itô's formula, we show that the condition (2) of the Lemma 3.1.

$$
\begin{aligned}
& L\left(-\frac{b_{1}^{l} A_{2}^{1-m}}{c_{2}^{u}} \frac{y^{1-m}}{1-m}\right) \leq \frac{b_{1}^{l}\left(A_{2}^{1-m}\right)^{l}}{c_{2}^{u}} y^{1-m}\left(r_{2}(t)-\frac{1}{T} \sum_{j=1}^{p} \ln \left(1+\beta_{j}\right)+\frac{m \sigma_{2}^{2}(t)}{2}\right) \\
&+\frac{b_{1}^{l} b_{2}^{l}}{c_{2}^{u}}\left(A_{2}^{2-m}\right)^{l} y^{2-m}-\frac{b_{1}^{l} A_{1}^{l} x}{1+A_{1}^{u} x} . \\
& L(-\ln x) \leq-\left(r_{1}(t)+\frac{1}{T} \sum_{j=1}^{p} \ln \left(1+\alpha_{j}\right)-\frac{\sigma_{1}^{2}(t)}{2}\right)+b_{1}^{u} A_{1}^{u} x+c_{1}^{u}\left(A_{2}^{m}\right)^{u} y^{m} .
\end{aligned}
$$

Let $\hat{V}=-\frac{b_{1}^{l}\left(A_{2}^{1-m}\right)^{l}}{c_{2}^{u}} \frac{y^{1-m}}{1-m}-\ln x$, then 


$$
\begin{aligned}
L(\hat{V}) \leq & \frac{b_{1}^{l}\left(A_{2}^{1-m}\right)^{l}}{c_{2}^{u}} y^{1-m}\left(r_{2}(t)-\frac{1}{T} \sum_{j=1}^{p} \ln \left(1+\beta_{j}\right)+\frac{m \sigma_{2}^{2}(t)}{2}\right) \\
& -\left(r_{1}(t)+\frac{1}{T} \sum_{j=1}^{p} \ln \left(1+\alpha_{j}\right)-\frac{\sigma_{1}^{2}(t)}{2}\right) \\
& +\frac{b_{1}^{l} b_{2}^{l}}{c_{2}^{u}}\left(A_{2}^{2-m}\right)^{l} y^{2-m}+b_{1}^{u} A_{1}^{u} x+c_{1}^{u}\left(A_{2}^{m}\right)^{u} y^{m} .
\end{aligned}
$$

By Young inequality, there exists a positive constant $H$ such that

$$
L(\hat{V}) \leq H\left(A_{2}^{2}\right)^{l} y^{2}+b_{1}^{u} A_{1}^{u} x-\left(r_{1}(t)+\frac{1}{T} \sum_{j=1}^{p} \ln \left(1+\alpha_{j}\right)-\frac{\sigma_{1}^{2}(t)}{2}\right) .
$$

Then

$$
\begin{aligned}
L\left(\hat{V}+\frac{H A_{2}^{u}}{b_{2}^{l}} y\right) \leq & b_{1}^{u} A_{1}^{u} x-\left(r_{1}(t)+\frac{1}{T} \sum_{j=1}^{p} \ln \left(1+\alpha_{j}\right)-\frac{\sigma_{1}^{2}(t)}{2}\right) \\
& +\frac{H A_{2}^{u}}{b_{2}^{l}} \frac{1}{T} \sum_{j=1}^{p} \ln \left(1+\beta_{j}\right) y+\frac{H}{b_{2}^{l}} c_{2}^{u} A_{1}^{u}\left(A_{2}^{m}\right)^{u} x y^{m} .
\end{aligned}
$$

Therefore,

$$
\begin{aligned}
& L V_{5}(x, y) \\
& \leq M_{1}\left[b_{1}^{u} A_{1}^{u} x-\left(r_{1}(t)+\frac{1}{T} \sum_{j=1}^{p} \ln \left(1+\alpha_{j}\right)-\frac{\sigma_{1}^{2}(t)}{2}\right)+\frac{H A_{2}^{u}}{b_{2}^{l} T} \sum_{j=1}^{p} \ln \left(1+\beta_{j}\right) y\right] \\
& +\frac{H}{b_{2}^{l}} c_{2}^{u} A_{1}^{u}\left(A_{2}^{m}\right)^{u} x y^{m}+\left(r_{2}(t)-\frac{1}{T} \sum_{j=1}^{p} \ln \left(1+\beta_{j}\right)+\frac{\sigma_{2}^{2}(t)}{2}\right)-b_{2}^{u} A_{2}^{u} y \\
& -b_{1}^{l} A_{1}^{l} x^{2}+A_{1}^{u} x\left[r_{1}^{u}+\frac{1}{T} \sum_{j=1}^{p} \ln \left(1+\alpha_{j}\right)\right]-\frac{c_{1}^{l} A_{2}^{l}}{c_{2}^{u}} y\left[r_{2}^{l}+b_{2}^{l} A_{2}^{l} y\right] . \\
& L V_{4}(t, x, y) \\
& =L V_{5}(x, y)+L V_{6}(t) \\
& \leq M_{1}\left[b_{1}^{u} A_{1}^{u} x+\frac{H A_{2}^{u}}{b_{2}^{l}} \frac{1}{T} \sum_{j=1}^{p} \ln \left(1+\beta_{j}\right) y+\frac{H}{b_{2}^{l}} c_{2}^{u} A_{1}^{u}\left(A_{2}^{m}\right)^{u} x y^{m}\right]-b_{1}^{l} A_{1}^{l} x^{2} \\
& \quad+b_{2}^{u} A_{2}^{u} y+A_{1}^{u} x\left[r_{1}^{u}+\frac{1}{T} \sum_{j=1}^{p} \ln \left(1+\alpha_{j}\right)\right]-\frac{c_{1}^{l} A_{2}^{l}}{c_{2}^{u}} y\left[r_{2}^{l}+b_{2}^{l} A_{2}^{l} y\right]-M_{1} \lambda_{1} \\
& \leq M_{1}\left[\frac{H}{b_{2}^{l}} c_{2}^{u} A_{1}^{u}\left(A_{2}^{u}\right)^{m} x y^{m}-\lambda_{1}\right]-\frac{b_{1}^{l} A_{1}^{l} x^{2}}{2}-\frac{c_{1}^{l}\left(A_{2}^{l}\right)^{2}}{2 c_{2}^{u}} b_{2}^{l} y^{2}+Q .
\end{aligned}
$$

where

$$
\begin{aligned}
Q= & \sup _{(x, y) \in R_{+}^{2}}\left\{M_{1} b_{1}^{u} A_{1}^{u} x+b_{2}^{u} A_{2}^{u} y+A_{1}^{u} x\left[r_{1}^{u}+\frac{1}{T} \sum_{j=1}^{p} \ln \left(1+\alpha_{j}\right)\right]-\frac{b_{1}^{l} A_{1}^{l} x^{2}}{2}\right. \\
& \left.-\frac{c_{1}^{l}\left(A_{2}^{l}\right)^{2}}{2 c_{2}^{u}} b_{2}^{l} y^{2}-\frac{c_{1}^{l} r_{2}^{l} A_{2}^{l}}{c_{2}^{u}} y+\frac{M_{1} H A_{2}^{u}}{b_{2}^{l}} \frac{1}{T} \sum_{j=1}^{p} \ln \left(1+\beta_{j}\right) y\right\}
\end{aligned}
$$


Let $M_{1}=\frac{2+Q}{\lambda_{1}}$, then $-\lambda_{1} M_{1}+Q=-2$. We choose a sufficiently small constant $\delta$ such that

$$
\begin{gathered}
0<\delta \leq\left\{\frac{\lambda_{1}}{4}, \frac{c_{1}^{l}\left(A_{2}^{l}\right)^{2} b_{2}^{l}}{4 c_{2}^{u} M_{1}}, \frac{b_{1}^{l} A_{1}^{l}}{4 M_{1}}\right\}, \\
-M_{1} \lambda_{1}-\frac{b_{1}^{l} A_{1}^{l}}{4 \delta^{2}}+Q_{1} \leq-1, \\
-M_{1} \lambda_{1}-\frac{c_{1}^{l}\left(A_{2}^{l}\right)^{2} b_{2}^{l}}{4 \delta^{2} c_{2}^{u}}+Q_{2} \leq-1 .
\end{gathered}
$$

Here

$$
\begin{aligned}
& Q_{1}=-\frac{b_{1}^{l} A_{1}^{l} x^{2}}{4}-\frac{c_{1}^{l}\left(A_{2}^{l}\right)^{2}}{2 c_{2}^{u}} b_{2}^{l} y^{2}+M_{1} \frac{H}{b_{2}^{l}} c_{2}^{u} A_{1}^{u}\left(A_{2}^{u}\right)^{m} x y^{m}+Q, \\
& Q_{2}=-\frac{b_{1}^{l} A_{1}^{l} x^{2}}{2}-\frac{c_{1}^{l}\left(A_{2}^{l}\right)^{2}}{4 c_{2}^{u}} b_{2}^{l} y^{2}+M_{1} \frac{H}{b_{2}^{l}} c_{2}^{u} A_{1}^{u}\left(A_{2}^{u}\right)^{m} x y^{m}+Q .
\end{aligned}
$$

The following proof is similar to the proof of $m=1$ and is omitted. This completes the proof.

\section{Extinction and Permanence of (1.4)}

In Section 3, we showed that under certain conditions, the system (1.4) has a periodic solution. Because in system (1.4), when $0<m<1$ the predator birth rate is of the form $\frac{c_{2} x(t)}{1+x(t)} y^{m-1}(t)$. Therefore, the predator birth rate goes to infinity when $y \rightarrow 0$, provided the prey population exists. So in this section, we will show that if the noise is sufficiently large, the solutions to the associated stochastic model will become extinct with probability one when $m=1$.

Definition 4.1 [15]. Let $x(t)$ be a solution to system (1.4).

1) If $\lim _{t \rightarrow \infty} x(t)=0$ a.s., then species $x(t)$ is said to be extinct;

2) If $\lim _{t \rightarrow \infty} \frac{1}{t} \int_{0}^{t} x(s) \mathrm{d} s>0$ a.s., then species $x(t)$ is said to be persistent in the mean.

Theorem 4.1. For any initial value $\left(x_{0}, y_{0}\right) \in R_{+}^{2}$, the solution $X(t)=(x(t), y(t))$ of (3.2) obeys

$$
\begin{gathered}
\limsup _{t \rightarrow \infty} \frac{\ln x(t)}{t} \leq r_{1}(t)-\frac{\sigma_{1}^{2}(t)}{2}+\frac{1}{T} \sum_{j=1}^{p} \ln \left(1+\alpha_{j}\right)<0, \\
\limsup _{t \rightarrow \infty} \frac{\ln y(t)}{t} \leq-r_{2}(t)+c_{2}(t)-\frac{\sigma_{2}^{2}(t)}{2}+\frac{1}{T} \sum_{j=1}^{p} \ln \left(1+\beta_{j}\right)<0 .
\end{gathered}
$$

that is, the solution of (3.2) is extinct exponentially with probability.

Proof. For system (3.2), using the Itô's formula, we have

$$
\mathrm{d} \ln x \leq\left(r_{1}(t)+\frac{1}{T} \sum_{j=1}^{p} \ln \left(1+\alpha_{j}\right)-\frac{\sigma_{1}^{2}(t)}{2}\right) \mathrm{d} t+\sigma_{1}(t) \mathrm{d} B_{1}(t),
$$


and

$$
\mathrm{d} \ln y \leq\left(-r_{2}(t)+\frac{1}{T} \sum_{j=1}^{p} \ln \left(1+\beta_{j}\right)+c_{2}(t)-\frac{\sigma_{2}^{2}(t)}{2}\right) \mathrm{d} t+\sigma_{2}(t) \mathrm{d} B_{2}(t) .
$$

Integrate both sides from 0 to $t$, then we get

$$
\begin{aligned}
& \frac{\ln x(t)-\ln x(0)}{t} \leq \frac{1}{t} \int_{0}^{t}\left(r_{1}(s)+\frac{1}{T} \sum_{j=1}^{p} \ln \left(1+\alpha_{j}\right)-\frac{\sigma_{1}^{2}(s)}{2}\right) \mathrm{d} s+\frac{1}{t} \int_{0}^{t} \sigma_{1}(t) \mathrm{d} B_{1}(t), \\
& \frac{\ln y(t)-\ln y(0)}{t} \\
& \leq \frac{1}{t} \int_{0}^{t}\left(-r_{2}(s)+\frac{1}{T} \sum_{j=1}^{p} \ln \left(1+\beta_{j}\right)+c_{2}(s)-\frac{\sigma_{2}^{2}(s)}{2}\right) \mathrm{d} s+\frac{1}{t} \int_{0}^{t} \sigma_{2}(t) \mathrm{d} B_{2}(t) .
\end{aligned}
$$

Let $P_{i}(t)=\int_{0}^{t} \sigma_{i}(t) \mathrm{d} B_{i}(t), i=1,2$, then $P_{i}(t)$ is a local martingale, and $\int_{0}^{t}\left(\sigma_{i}^{2}\right)^{u} \mathrm{~d} s \leq\left(\sigma_{i}^{2}\right)^{u} t$.

By the strong law of martingale, we have

$$
\lim _{t \rightarrow \infty} \frac{P_{i}(t)}{t}=0 \text { a.s.. }
$$

Hence we can derive

$$
\begin{gathered}
\lim _{t \rightarrow \infty} \sup \frac{\ln x(t)}{t} \leq r_{1}(t)-\frac{\sigma_{1}^{2}(t)}{2}+\frac{1}{T} \sum_{j=1}^{p} \ln \left(1+\alpha_{j}\right)<0 \text { a.s. } \\
\limsup _{t \rightarrow \infty} \frac{\ln y(t)}{t} \leq-r_{2}(t)+\frac{1}{T} \sum_{j=1}^{p} \ln \left(1+\beta_{j}\right)+c_{2}(t)-\frac{\sigma_{2}^{2}(t)}{2}<0 \text { a.s. }
\end{gathered}
$$

Thus, $\lim _{t \rightarrow \infty} x(t)=0, \lim _{t \rightarrow \infty} y(t)=0$ a.s.

Theorem 4.2. Assume

$$
\begin{gathered}
\int_{0}^{t}\left(r_{1}(s)-\frac{\sigma_{1}^{2}(s)}{2}\right) \mathrm{d} s+\frac{1}{T} \sum_{j=1}^{p} \ln \left(1+\alpha_{j}\right)>0, \\
\int_{0}^{t}\left(-r_{2}(s)+c_{2}(s)-\frac{\sigma_{2}^{2}(s)}{2}\right) \mathrm{d} s+\frac{1}{T} \sum_{j=1}^{p} \ln \left(1+\beta_{j}\right)<0 .
\end{gathered}
$$

then the predators of system (3.2) will eventually extinction, and prey populations go to persistent.

Proof. By Theorem 4.1, we have

when $\int_{0}^{t}\left(-r_{2}(s)+c_{2}(s)-\frac{\sigma_{2}^{2}(s)}{2}\right) \mathrm{d} s+\frac{1}{T} \sum_{j=1}^{p} \ln \left(1+\beta_{j}\right)<0$, then $\lim _{t \rightarrow \infty} y(t)=0$ a.s.

So predators will eventually extinction. Next, we prove the persistence of prey quantity.

For system (3.2), by Itô's formula (3.2), we have 


$$
\begin{aligned}
\mathrm{d} \ln x= & \left(r_{1}(t)+\frac{1}{T} \sum_{j=1}^{p} \ln \left(1+\alpha_{j}\right)-b_{1}(t) A_{1}(t) x-\frac{c_{1}(t) y A_{2}(t)}{1+x A_{1}(t)}-\frac{\sigma_{1}^{2}(t)}{2}\right) \mathrm{d} t \\
& +\sigma_{1}(t) \mathrm{d} B_{1}(t) .
\end{aligned}
$$

Integrate both sides of this equation from 0 to $t$ and divide by $t$, then

$$
\begin{aligned}
& \frac{\ln x(t)-\ln x(0)}{t} \\
& =\frac{1}{t} \int_{0}^{t}\left(r_{1}(s)+\frac{1}{T} \sum_{j=1}^{p} \ln \left(1+\alpha_{j}\right)-\frac{\sigma_{1}^{2}(s)}{2}\right) \mathrm{d} s-\frac{1}{t} \int_{0}^{t} b_{1}(s) A_{1}(s) x \mathrm{~d} s \\
& -\frac{1}{t} \int_{0}^{t} \frac{c_{1}(s) y A_{2}(s)}{1+x A_{1}(s)} \mathrm{d} s+\frac{1}{t} \int_{0}^{t} \sigma_{1}(t) \mathrm{d} B_{1}(t) .
\end{aligned}
$$

According to the periodicity of $r_{1}(t), \sigma_{1}^{2}(t)$, we get

$$
\frac{1}{t} \int_{0}^{t}\left(r_{1}(s)-\frac{\sigma_{1}^{2}(s)}{2}\right) \mathrm{d} s=\frac{1}{T} \int_{0}^{t}\left(r_{1}(s)-\frac{\sigma_{1}^{2}(s)}{2}\right) \mathrm{d} s .
$$

By Theorem 4.1, we have

$$
\lim _{t \rightarrow \infty} \frac{\int_{0}^{t} \sigma_{1}(t) \mathrm{d} B_{1}(t)}{t}=0 \text { a.s. }
$$

Suppose $y(t)$ is bounded on $t$, and $\lim _{t \rightarrow \infty} y(t)=0$ a.s., then

$$
\lim _{t \rightarrow \infty} \frac{1}{t} \int_{0}^{t} \frac{c_{1}(s) y(s) A_{2}(s)}{1+x A_{1}(s)} \mathrm{d} s=0 .
$$

Then, Equation (4.1) can be changed to

$$
0=\frac{1}{T} \int_{0}^{t}\left(r_{1}(s)-\frac{\sigma_{1}^{2}(s)}{2}\right) \mathrm{d} s+\frac{1}{T} \sum_{j=1}^{p} \ln \left(1+\alpha_{j}\right)-\lim _{t \rightarrow \infty} \frac{1}{t} \int_{0}^{t} b_{1}(s) A_{1}(s) x \mathrm{~d} s .
$$

So

$$
b_{1}^{u} \lim _{t \rightarrow \infty} \frac{1}{t} \int_{0}^{t} x(s) \mathrm{d} s \geq \frac{1}{T} \int_{0}^{t}\left(r_{1}(s)-\frac{\sigma_{1}^{2}(s)}{2}\right) \mathrm{d} s+\frac{1}{T} \sum_{j=1}^{p} \ln \left(1+\alpha_{j}\right)>0 .
$$

which shows the number of prey is persistent. The proof is completed.

\section{Numerical Simulation}

In this section, by using the Higham [16] simulation method, we illustrate our main results. The red and blue lines represent predators and prey, respectively.

Example 1. Let $r_{1}(t)=0.7, \quad m=1, \quad b_{1}(t)=0.55, \quad r_{2}(t)=0.09$, $b_{2}(t)=0.15, \quad c_{1}(t)=c_{2}(t)=0.35, \quad \sigma_{1}=0.03, \quad \sigma_{2}=0.05, \quad p=1$, $\alpha=\beta=0.01, \quad T=2 \pi$, and initial value $(x(0), y(0))=(1,0.7)$. Thus, $\lambda \approx 0.21>0$,

$\int_{0}^{2 \pi}\left(r_{1}(t)-\frac{\sigma_{1}^{2}(t)}{2}\right) \mathrm{d} t+\frac{1}{T} \sum_{j=1}^{p} \ln \left(1+\alpha_{j}\right) \approx 4.4>0$. From Theorems 3.1, we know that system (1.4) has a periodic solution. We use Figure 1 to illustrate this result (see Figure 1). 

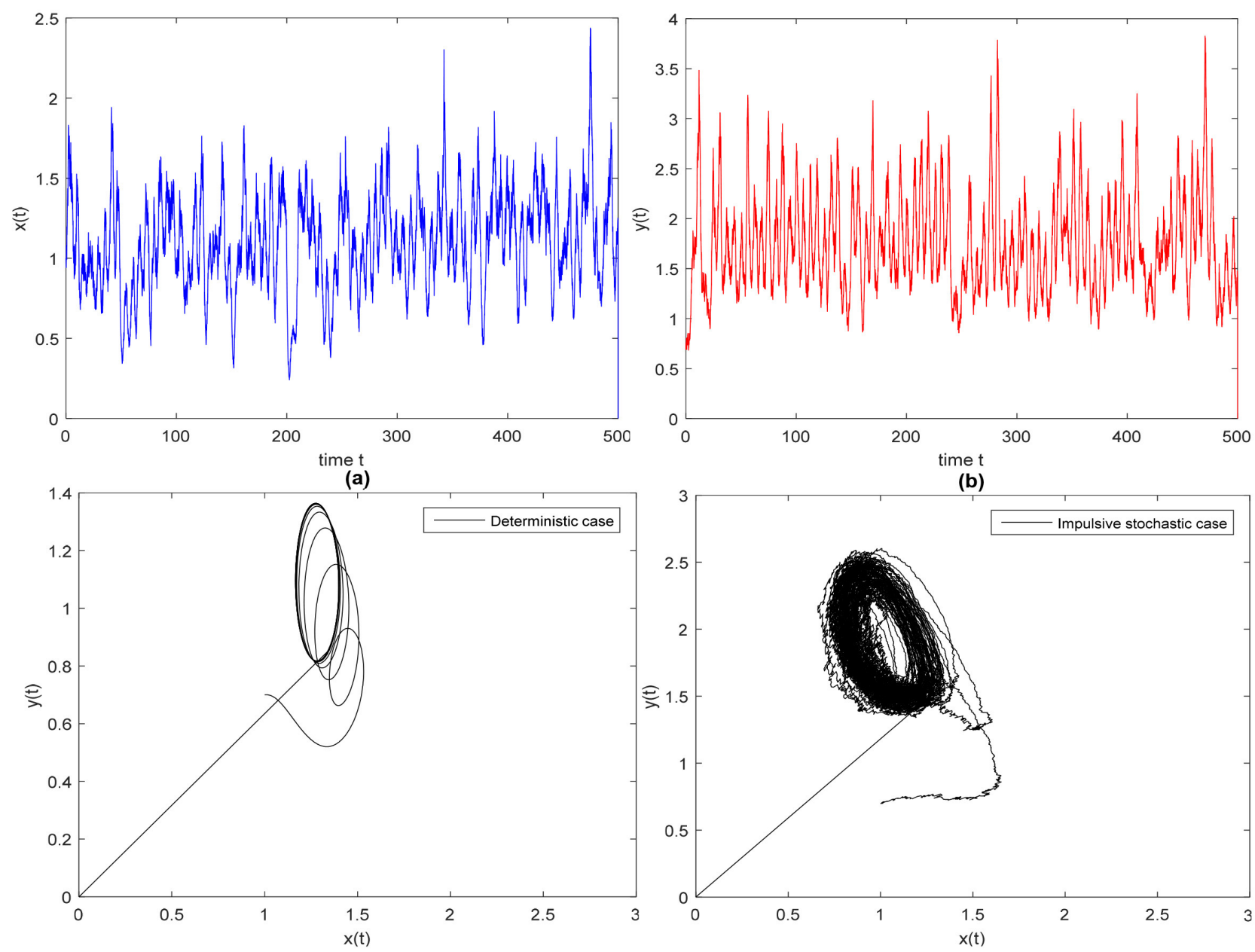

(b)

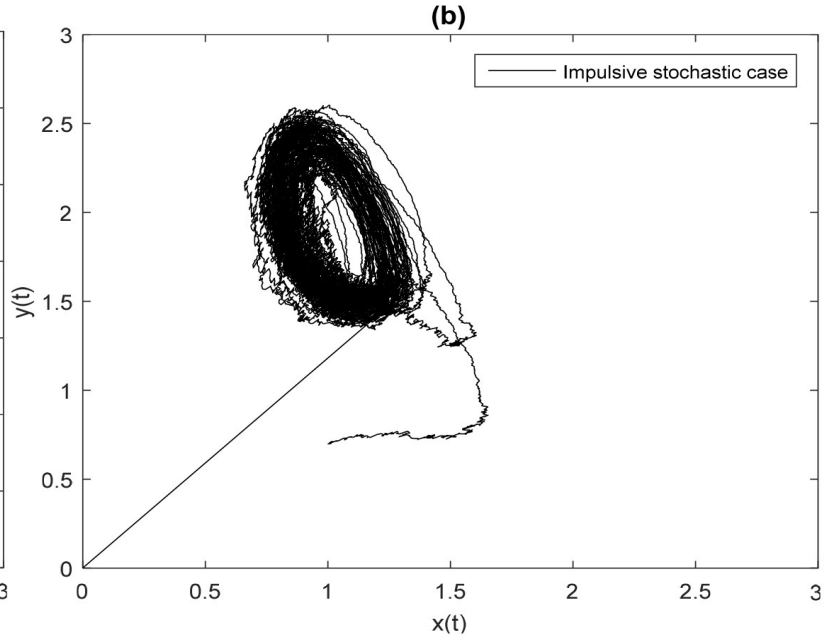

(c)

(d)

Figue 1. The solutions of (1.4) with smaller white noise, $\sigma_{1}=0.03, \sigma_{2}=0.05$, (a) the prey is persistent, (b) the predator is persistent, (c) and (d) represent distribution scatter of (1.4).

Example 2. When we let $\sigma_{1}=0.82, \sigma_{2}=0.85$, and the other parameters are the same as Example 1. we have $\int_{0}^{2 \pi}\left(r_{1}(t)-\frac{\sigma_{1}^{2}(t)}{2}\right) \mathrm{d} t+\frac{1}{T} \sum_{j=1}^{p} \ln \left(1+\alpha_{j}\right) \approx 3.42>0$, $\int_{0}^{T}\left(-r_{2}(t)+c_{2}(t)-\frac{\sigma_{2}^{2}(t)}{2}\right) \mathrm{d} s+\frac{1}{T} \sum_{j=1}^{p} \ln \left(1+\beta_{j}\right) \approx-4.23<0$, then Theorem 3.1 and 4.2 tell us that big white noise intensity can cause the predators extinction. We use Figure 2 to explain these results (see Figure 2).

Example 3. Set $r_{1}(t)=0.5, \quad b_{1}(t)=0.45, \quad r_{2}(t)=0.5, \quad b_{2}(t)=0.4$, $c_{1}(t)=0.45, p=1, \quad c_{2}(t)=0.35, \sigma_{1}(t)=1.2, \sigma_{2}(t)=0.85, \alpha=\beta=0.3$, $m=1, T=2 \pi$, Thus, we have $\int_{0}^{2 \pi}\left(r_{1}(t)-\frac{\sigma_{1}^{2}(t)}{2}\right) \mathrm{d} t+\frac{1}{T} \sum_{j=1}^{p} \ln \left(1+\alpha_{j}\right) \approx-0.01>0$, $\int_{0}^{T}\left(-r_{2}(t)+c_{2}(t)-\frac{\sigma_{2}^{2}(t)}{2}\right) \mathrm{d} s+\frac{1}{T} \sum_{j=1}^{p} \ln \left(1+\beta_{j}\right) \approx-0.1<0$. By Theorems 4.1, we can determine that the two species will become extinct (see Figure 3). 


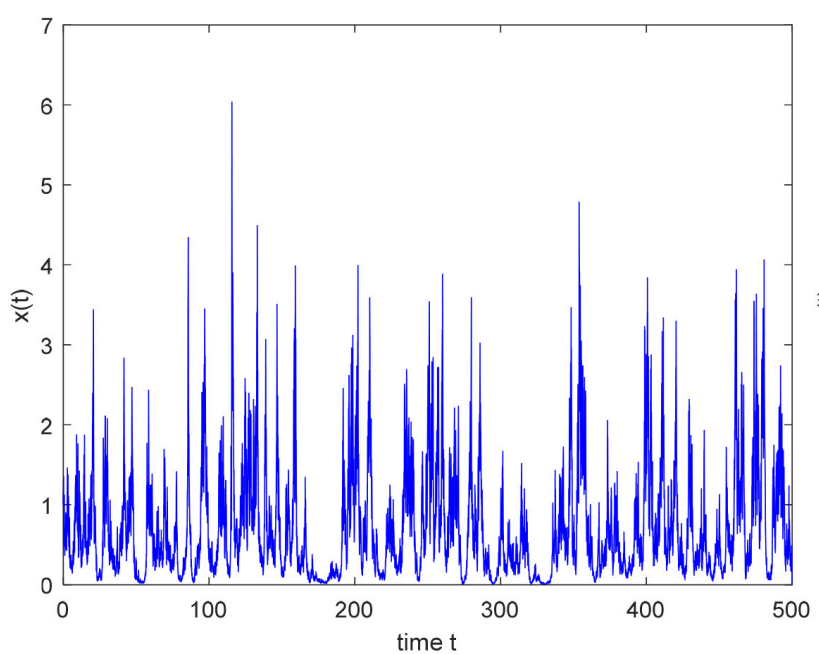

(a)

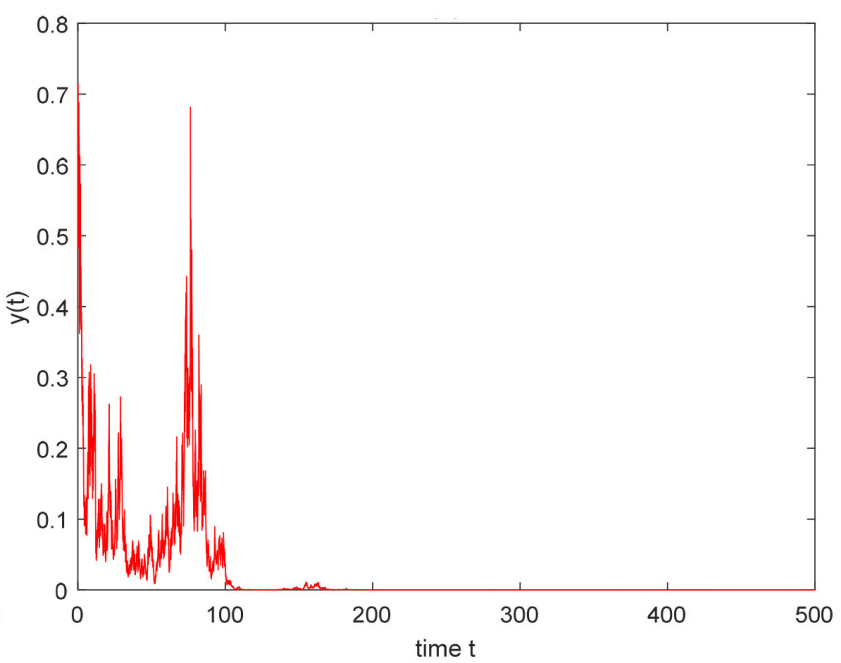

(b)

Figure 2. The solution of (1.4) with bigger white noise $\sigma_{1}=0.82, \sigma_{2}=0.85$. (a) the prey is persistent in the mean, (b) the predator is extinction.

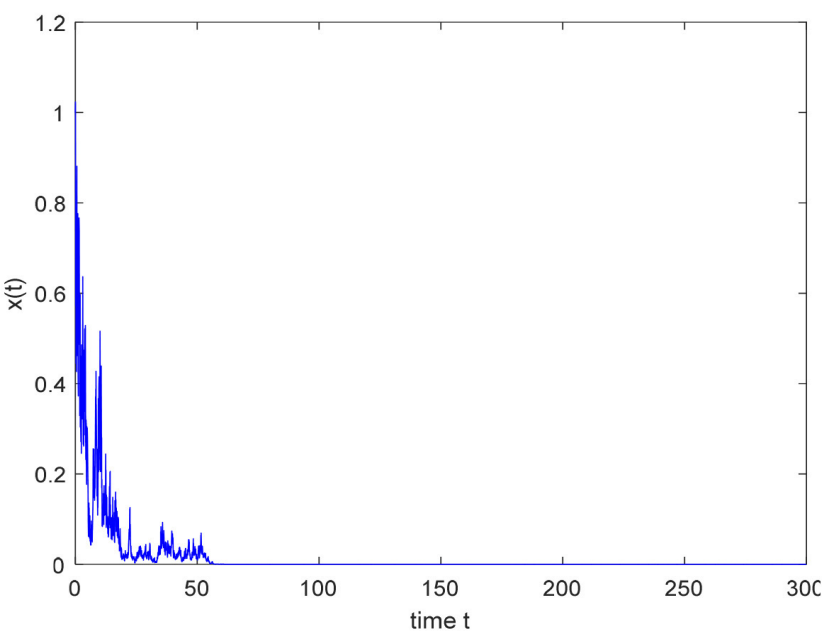

(a)

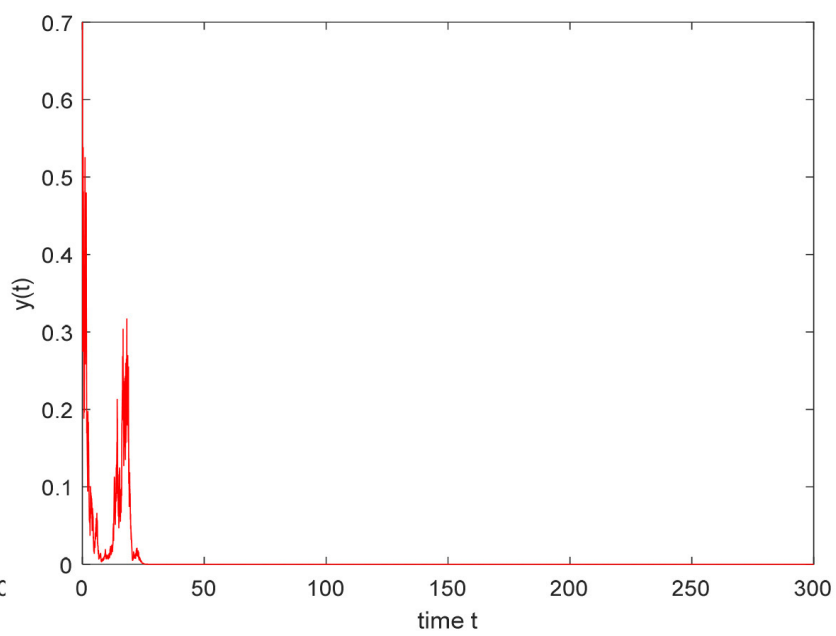

(b)

Figure 3. (a), (b): The initial value is $(x(0), y(0))=(1,0.7)$ in system (1.4). Two species will become extinct.

\section{Conclusions}

In this paper, we propose a predator-prey model with impulsive disturbance in a random environment. We prove that system (1.4) has a globally unique positive solution and a positive periodic solution. In this article, our results are as follows:

a) The effects of white noise and impulse interference on the model are considered.

b) If the impulses are large enough such that the conditions of $\lambda, \lambda_{1}$ hold, then system (1.4) has a positive $T$-periodic solution.

c) The main results reveal that large white noise will force the population to become extinct while the population may be persistent under a relatively small 
white noise.

\section{Acknowledgements}

This work was supported by the National Natural Science Foundation of China (11861027) and Natural Science Foundation of Guangxi (2016 GXNSFAA380194).

\section{Conflicts of Interest}

The authors declare no conflicts of interest regarding the publication of this paper.

\section{References}

[1] Lv, J.L. and Wang, K. (2011) Asymptotic Properties of a Stochastic Predator-Prey System with Holling II Functional Response. Communications in Nonlinear Science and Numerical Simulation, 16, 4037-4048.

https://doi.org/10.1016/j.cnsns.2011.01.015

[2] Li, Y. and Gao, H. (2008) Existence, Uniqueness and Global Asymptotic Stability of Positive Solutions of a Predator-Prey System with Holling II Functional Response with Random Perturbation. Nonlinear Analysis, 68, 1694-1705.

https://doi.org/10.1016/j.na.2007.01.008

[3] Hassell, M. (1975) Density Dependence in Single-Species Population. Journal of Animal Ecology, 44, 283-295. https://doi.org/10.2307/3863

[4] Hassell, M. (1971) Mutual Interference between Searching Insect Parasites. Journal of Animal Ecology, 40, 473-486. https://doi.org/10.2307/3256

[5] Liu, Q., Zu, L. and Jiang, D.Q. (2016) Dynamics of Stochastic Predator-Prey Models with Holling II Functional Response. Communications in Nonlinear Science and Numerical Simulation, 37, 62-76. https://doi.org/10.1016/j.cnsns.2016.01.005

[6] Wang, K. and Zhu, Y. (2013) Periodic Solutions, Permanence and Global Attractivity of a Delayed Impulsive Prey-Predator System with Mutual Interference. Nonlinear Analysis: Real World Applications, 14, 1044-1054.

https://doi.org/10.1016/j.nonrwa.2012.08.016

[7] Lv, Y. and Du, Z. (2011) Existence and Global Attractivity of a Positive Periodic Solution to a Lotka-Volterra Model with Mutual Interference and Holling III Type Functional Response. Nonlinear Analysis. Real World Applications, 12, 3654-3664. https://doi.org/10.1016/j.nonrwa.2011.06.022

[8] Mandal, P. and Bnerjee, M. (2012) Stochastic Persistence and Stationary Distribution in a Holling-Tanner Type Prey-Predator Model. Physica A, 391, 1216-1233. https://doi.org/10.1016/j.physa.2011.10.019

[9] Rudnicki, R. and Pichor, K. (2007) Influence of Stochastic Perturbation on Prey-Predator Systems. Mathematical Biosciences, 206, 108-119. https://doi.org/10.1016/j.mbs.2006.03.006

[10] Liu, Q. and Chen, Q. (2015) Analysis of a General Stochastic Non-Autonomous Logistic Model with Delays and Lévy Jumps. Journal of Mathematical Analysis and Applications, 433, 95-120. https://doi.org/10.1016/j.jmaa.2015.07.030

[11] Zuo, W.J. and Jiang, D.Q. (2016) Periodic Solutions for a Stochastic Non Autonomous Holling-Tanner Predator-Prey System with Impulses. Nonlinear Analysis: Hybrid Systems, 22, 191-201. https://doi.org/10.1016/j.nahs.2016.03.004 
[12] Li, D. and Xu, D. (2013) Periodic Solutions of Stochastic Delay Differential Equations and Applications to Logistic Equation and Neural Networks. Korean Mathematical Society, 50, 1165-1181. https://doi.org/10.4134/JKMS.2013.50.6.1165

[13] Khasminskii, R. (2011) Stochastic Stability of Differential Equations. Springer, Berlin. https://doi.org/10.1007/978-3-642-23280-0

[14] Wang, H.F. and Hu, Z.X. (2018) Analysis of a Random Model of Prey-Prey with Impulse. Journal of Henan University (Natural Science Edition), 48, 475-484.

[15] Wu, R.H., Zou, X.L. and Wang, K. (2015) Asymptotic Behavior of a Stochastic Non-Autonomous Predator-Prey Model with Impulsive Perturbations. Communications in Nonlinear Science and Numerical Simulation, 20, 965-974.

https://doi.org/10.1016/j.cnsns.2014.06.023

[16] Higham, D. (2001) An Algorithmic Introduction to Numerical Simulation of Stochastic Differential Equations. SIAM Review, 43, 525-546.

https://doi.org/10.1137/S0036144500378302 